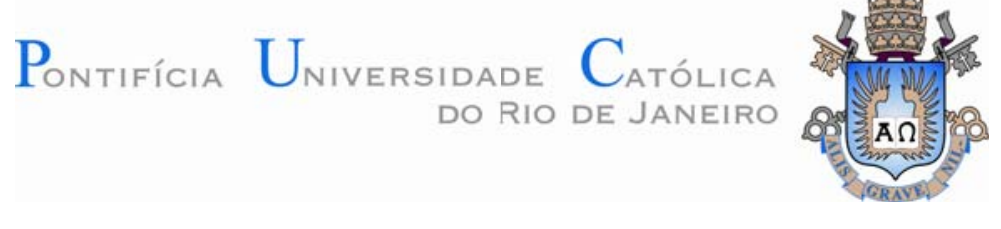

Ranena Verónica Ponce Flores

\title{
Modelagem em Duas Escalas do Acoplamento de Fenômenos Eletro-Químico-Mecânicos em Polímeros Expansivos Sensíveis a pH e Salinidade
}

Tese de Doutorado

Tese apresentada ao Programa de Pós-graduação em Engenharia Mecânica do Departamento de Engenharia Mecânica da PUCRio como requisito parcial para obtenção do título de Doutor em Engenharia Mecânica.

Orientador : Arthur Martins Barbosa Braga Co-Orientador: Márcio Arab Murad Co-Orientador: $\quad$ Sidarta Araújo de Lima 


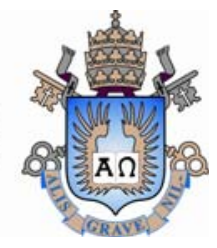

Ranena Verónica Ponce Flores

\section{Modelagem em Duas Escalas do Acoplamento de Fenômenos Eletro-Químico-Mecânicos em Polímeros Expansivos Sensíveis a pH e Salinidade}

Tese apresentada ao Programa de Pós-graduação em Engenharia Mecânica do Departamento de Engenharia Mecânica do Centro Técnico Científico da PUC-Rio como requisito parcial para obtenção do título de Doutor em Engenharia Mecânica. Aprovada pela Comissão examinadora abaixo assinada.

Prof. Arthur Martins Barbosa Braga

Orientador

Departamento de Engenharia Mecânica - PUC-Rio

Prof. Márcio Arab Murad

Co-Orientador

Coordenação de Mecânica Computacional — LNCC

Prof. Sidarta Araújo de Lima

Co-Orientador

Departamento de Tecnologias e Linguagens - UFRRJ

Prof. Lavinia Maria Sanabio Alves Borges

Programa de Engenharia Mecânica - UFRJ

Prof. Marcelo Amorim Savi

Programa de Engenharia Mecânica - UFRJ

Prof. Márcio da Silveira Carvalho Departamento de Engenharia Mecânica - PUC-Rio

Prof. Norbert Fritz Miekeley Departamento de Química - PUC-Rio

Prof. José Eugênio Leal Coordenador Setorial do Centro Técnico Científico - PUC-Rio 
Todos os direitos reservados. É proibida a reprodução total ou parcial do trabalho sem autorização da universidade, do autor e do orientador.

Ranena Verónica Ponce Flores

Graduou-se em Engenharia Química na USB (Caracas, Venezuela) em 1999. Trabalhou em PDVSA-Intevep no departamento de Recuperação Melhorada de Petróleo, onde recebeu bolsa para cursar estudos de Especialização em Engenharia de Reservatórios na USB em 2002. Atualmente trabalha no Laboratório de Microhidrodinâmica e Escoamento em Meios Porosos na PUC-Rio como Coordenador de Projetos de Pesquisa na área de escoamento de emulsões em meios porosos.

Ficha Catalográfica

Ponce Flores, Ranena Verónica

Modelagem em Duas Escalas do Acoplamento de Fenômenos Eletro-Químico-Mecânicos em Polímeros Expansivos Sensíveis a pH e Salinidade / Ranena Verónica Ponce Flores; orientador: Arthur Martins Barbosa Braga; coorientadores: Márcio Arab Murad, Sidarta Araújo de Lima. 2008.

159 f.: il. ; $29,7 \mathrm{~cm}$

1. Tese (Doutorado em Engenharia Mecânica) - Pontifícia Universidade Católica do Rio de Janeiro, Rio de Janeiro, 2008.

Inclui bibliografia

1. Engenharia Mecânica - Teses. 2. Polímeros Sensíveis ao pH. 3. Hidrogéis. 4. Acoplamento Electro-Químico-Mecânico em Polímeros. 5. Homogeneização. 6. Elementos Finitos. I. Braga Barbosa, Arthur Martins. II. Murad Arab, Márcio. III. Lima, Sidarta Araújo de. IV. Pontifícia Universidade Católica do Rio de Janeiro. Departamento de Engenharia Mecânica. V. Título. 
Dedico este trabalho aos meus amados pais Gladys e Jesús, a eles esta e todas as minhas conquistas. 


\section{Agradecimentos}

A DEUS, por me conceder coragem, perseverança e sabedoria, para o feliz término do presente trabalho.

Às benções da minha vida: meus pais Gladys e Jesús, e Felicle, e aos meus familiares e amigos que de longe me encorajaram em todo momento.

Aos meus orientadores, professores Arthur Braga, Márcio Murad e Sidarta Lima, pela paciência e imensurável apoio e dedicação na direção do presente trabalho; meu profundo agradecimento pelo privilégio de aprender de sua experiência e pela generosidade de compartilhar comigo parte do seu valioso conhecimento.

Ao Vladimir pela inestimável amizade e sempre acertados conselhos.

Ao meu chefe, professor Márcio Carvalho, pela paciência, compreensão e apoio em momentos difíceis e ao professor Hans pelo incentivo e amizade.

Aos membros da banca examinadora pela disposição para analisar o presente trabalho bem como pelas sugestões recebidas para enriquecê-lo.

Ao professor Norbert Miekeley (PUC-Rio) pelo incondicional apoio experimental, fundamental para a realização deste trabalho. Faço extensivo o meu agradecimento à professora Judith Felcman bem como ao Álvaro, Heloisa, Maurício, Pedro e Guelintong pela ajuda e simpatia.

Aos professores Marcos Lopes e Elizabeth Monteiro (IMA), ao professor Marco Cremona (PUC-Rio/INMETRO) e à professora Sonia Louro (PUCRio) pelas relevantes sugestões e apoio experimental.

Ao professor Silvio Soriano (CEFETQ), que de forma fraterna me cedeu seu tempo e paciência para repensar em química analítica aplicada a polímeros.

Aos meus colegas e amigos da PUC-Rio André, Fabio, Eduardo, Frank, Epifanio, Sully, Carla, Paula, Carolina, Oscar, Luciana, Roberth, Alex, Priscilla, Christiano e Angélica pelo incentivo e convívio durante todos estes anos. Aos meus colegas do LNCC Maicon e Marcos bem como às professoras Sandra Malta e Regina Almeida pelo apoio e gentileza em todo momento.

A todo o pessoal acadêmico, técnico e administrativo do Departamento de Engenharia Mecânica da PUC-Rio, bem como a todos os que não menciono, e que direta ou indiretamente, contribuíram na realização deste trabalho.

À PUC-Rio, pela magnífica oportunidade de crescer academicamente, e ao CNPq pelo auxílio financeiro. 


\section{Resumo}

Ponce Flores, Ranena Verónica; Braga Barbosa, Arthur Martins; Murad Arab, Márcio; Lima, Sidarta Araújo de. Modelagem em Duas Escalas do Acoplamento de Fenômenos Eletro-Químico-Mecânicos em Polímeros Expansivos Sensíveis a pH e Salinidade. Rio de Janeiro, 2008. 159p. Tese de Doutorado - Departamento de Engenharia Mecânica, Pontifícia Universidade Católica do Rio de Janeiro.

Nas últimas décadas, tem se desenvolvido um crescente interesse em polímeros de troca iônica capazes de responder a diversos estímulos externos, sendo utilizados nos mais variados campos da ciência como sensores, transdutores e atuadores. Este tipo de materiais em contato com soluções aquosas sofrem deformações decorrentes de mudanças de campos magnéticos, temperatura, $\mathrm{pH}$, campos elétricos e/ou força iônica. Sendo o pH um dos parâmetros operacionais críticos no controle de diferentes processos químicos, os polímeros sensíveis a $\mathrm{pH}$ constituem uma alternativa promissora em diversas áreas de pesquisa, e de particular interesse neste trabalho; na área de monitoramento do $\mathrm{pH}$ aplicado à industria do petróleo. O grande potencial e funcionabilidade deste tipo de polímeros tem despertado a necessidade de estudar e entender seu comportamento através de modelos matemáticos. O objetivo deste trabalho é desenvolver um modelo químico-eletro-mecânico para o estudo do inchamento de uma resina de troca iônica em equilíbrio com uma solução aquosa eletrolítica, como função das mudanças do pH e concentração salina da solução. Com o intuito de determinar parâmetros inerentes ao equilíbrio químico associados à densidade de carga elétrica superficial da resina, foram conduzidos ensaios de titulação potenciométrica bem como medidas de inchamento para três concentrações de $\mathrm{NaCl}$. Objetivando investigar a microestrutura e composição do polímero foram realizados ensaios de Microscopia Eletrônica de Varredura (MEV) e Espectroscopia de Infravermelho por Transformada de Fourier (FTIV). A modelagem matemática foi desenvolvida em duas escalas (micro-macro) adotando a técnica de homogeneização de estruturas periódicas. Para a modelagem do inchamento da resina foi assumida a hipótese de material linearmente elástico, incorporando os fenômenos eletro-osmóticos na escala local mediante a solução do problema de Poisson-Boltzmann e do balanço de momento linear no fluido. O sistema de equações não lineares postos na escala macroscópica foi discretizado através do Método de Elementos Finitos (Galerkin) e resolvido em forma acoplada mediante o método de Newton-Raphson. Os resultados teóricos mostram a existência de dois regimes de inchamento na resina: um regime dominado pela densidade de carga elétrica na superfície do sólido e um segundo regime onde o inchamento da resina é puramente governado pela força iônica da solução eletrolítica.

\section{Palavras-chave}

Polímeros Sensíveis ao pH. Hidrogéis. Acoplamento Electro-QuímicoMecânico em Polímeros. Homogeneização. Elementos Finitos. 


\section{Abstract}

Ponce Flores, Ranena Verónica; Braga Barbosa, Arthur Martins; Murad Arab, Márcio; Lima, Sidarta Araújo de. A Two-Scale Model for Coupled Electro-Chemo-Mechanical Phenomena in Expansive Polymers Sensitive to $\mathbf{p H}$ and Salinity. Rio de Janeiro, 2008. 159p. PhD Thesis - Departamento de Engenharia Mecânica, Pontifícia Universidade Católica do Rio de Janeiro.

In the last few decades, the interest in ion exchange polymers that are able to respond under several external stimuli has grown; these polymers are being used in the most diverse fields of science such as sensors, transducers and actuators. This type of material, when in contact with aqueous solutions, undergoes deformations caused by changes in magnetic fields, temperature, $\mathrm{pH}$, electrical fields and/or ionic strength. Since the $\mathrm{pH}$ is a critical operating parameter in the control of different chemical processes, $\mathrm{pH}$ sensitive polymers have become a promising alternative in several areas of research and of particular interest in this work in the area of $\mathrm{pH}$ monitoring applied to the oil industry. The great potential and functionality of this type of polymers has increased the need to study and understand their behavior through mathematical models. The purpose of this study was to develop a chemo-electromechanical model to study the swelling of an ion exchange resin in equilibrium with an electrolyte solution, as a function of changes in $\mathrm{pH}$ and saline concentration of the solution. In order to determine parameters related to the chemical equilibrium associated with the surface electric charge density of resin, potentiometric titrations as well as measures of swelling for three $\mathrm{NaCl}$ concentrations were developed. Aiming to investigate the microstructure and composition of the polymer, Scanning Electron Microscopy (SEM) and Fourier Transform Infrared Spectroscopy (FTIR) tests were performed. The mathematical modeling was developed in two scales (micromacro) adopting the homogenization technique of periodic structures. For modeling the swelling of the resin, the hypothesis of linear elastic material was assumed, incorporating the electroosmotic phenomena in the local scale through the solution to the Poisson-Boltzmann problem and the balance of linear momentum in the fluid. The system of nonlinear equations placed in the macroscopic scale was discretized through the Finite Element Method (Galerkin) and the coupled equations solved with the method of Newton-Raphson. The theoretical results show the presence of two swelling regimes in the resin: a regime controlled by the charge density on the surface of the solid and a secondary regime where the swelling of the resin is purely governed by the ionic strength of the electrolyte solution.

\section{Keywords}

1. pH Sensitive Polymers. 2. Hydrogels. 3. Electro-Chemo-Mechanical Coupling in Polymers. 4. Homogenization. 5. Finite Elements. 


\section{Sumário}

1 Introdução $\quad \mathbf{1 5}$

$\begin{array}{lll}1.1 & \text { Generalidades sobre Materiais Inteligentes } & 15\end{array}$

$\begin{array}{lll}1.2 & \text { Aplicações de resinas trocadoras de íons } & 16\end{array}$

1.3 Motivação 16

$\begin{array}{lll}1.4 & \text { Objetivo } & 17\end{array}$

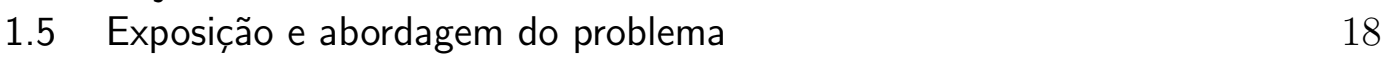

$\begin{array}{lll}1.6 & \text { Estado da Arte } & 19\end{array}$

1.7 Escopo e Roteiro da Tese 20

2 Eletroquímica na Nanoescala $\quad 21$

2.1 Trocadores iônicos 21

2.2 Resinas de troca iônica 26

2.2.1 Características de resinas catiônicas 30

2.2.2 Métodos de estudo 33

2.2.3 Teoria sobre o inchamento e mecanismo de troca 37

2.3 Equilíbrio químico 40

2.3.1 Descrição da carga elétrica 42

3 Resultados Experimentais $\quad 45$

3.1 Titulação potenciométrica 46

3.1.1 Titulação da resina 48

3.1.2 Determinação da capacidade de troca catiônica da resina 53

3.2 Determinação das constantes de equilíbrio químico 56

3.3 Investigação da composição da resina mediante espectroscopia de infravermelho por transformada de Fourier (FTIV) 62

3.4 Microestrutura da resina 66

3.5 Medida do inchamento 68

4 Modelagem em Duas Escalas $\quad 72$

4.1 Dupla Camada Elétrica 73

4.1.1 Teoria de Gouy-Chapman $\quad 75$

4.2 Modelo microscópico $\quad 78$

$\begin{array}{lll}\text { 4.2.1 Eletrostática } & 79\end{array}$

4.2.2 Problema de Equilíbrio no Fluido: $\quad 80$

4.2.3 Deformação do sólido $\quad 81$

4.2.4 Balanço de Massa no sólido $\quad 81$

4.2.5 Condições de contorno $\quad 82$

4.2.6 Resumo da modelagem microscópica $\quad 84$

4.3 Homogeneização $\quad 85$

4.3.1 Teoría de Homogeneização $\quad 86$

4.3.2 Homogeneização do modelo $\quad 88$

4.3.2.1 Adimensionalização $\quad 89$

4.3.2.2 Normalização $\quad 91$

4.3.2.3 Expansão Assintótica 93 
$\begin{array}{lll}\text { 4.3.2.4 Problemas de Fechamento } & 98\end{array}$

$\begin{array}{lll}\text { 4.3.2.5 Equações macroscópicas } & 99\end{array}$

4.3.2.6 Resumo do modelo em duas escalas 100

4.3.2.7 Particularização do modelo para placas planas paralelas 103

4.3.2.8 Resumo do Modelo de duas escalas unidimensional 108

5 Discretização do Modelo $\quad 110$

$\begin{array}{lll}5.1 & \text { Formulação Variacional } & 110\end{array}$

5.2 Método de Newton-Raphson 112

5.2.1 Aproximação por Elementos finitos: Método de Galerkin 113

5.2.2 Formulação Matricial 114

5.2.2.1 Particularização para o caso unidimensional 116

6 Modelagem Computacional 118

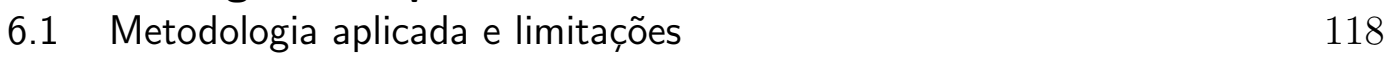

6.2 Simulações Numéricas $\quad 124$

6.2.1 Efeito das constantes de equilíbrio 126

6.2.2 Efeito do módulo de Young 128

6.2.3 Efeito da área superficial específica 129

$\begin{array}{ll}\text { 6.2.4 Efeito da espessura das placas } & 130\end{array}$

$\begin{array}{ll}\text { 6.2.5 Análise dos regimes de inchamento } & 132\end{array}$

7 Conclusões e Recomendações $\quad 136$

$\begin{array}{ll}\text { Referências Bibliográficas } & 138\end{array}$

A Apêndice $\quad 153$

A.1 Efeito de $K_{1} \quad 153$

$\begin{array}{lll}\text { A.2 Efeito de } K_{2} & 154\end{array}$

A.3 Efeito de $E_{s} \quad 155$

$\begin{array}{ll}\text { A.4 Efeito de } A s & 156\end{array}$

$\begin{array}{ll}\text { A.5 Efeito de } \delta & 157\end{array}$ 


\section{Lista de figuras}

2.1 Esquema ilustrativo de um trocador catiônico orgânico que intercambia íons $\mathrm{H}^{+}$por $\mathrm{Na}^{+}$em uma solução aquosa.

2.2 Estrutura de dois tipos de trocadores naturais: (a) Caulinita, (b) Carvão.

2.3 Estrutura esquemática de um polímero entrecruzado. Conformações em estado seco e inchado.

2.4 Grupos funcionais típicos em resinas de troca catiônica e aniônica. 28

2.5 Resinas em forma de pérolas. Resina catiônica (parte superior) e aniônica (parte inferior).

2.6 Representação esquemática do processo de polimerização do poli(ácido acrílico) (PAA) em presença do divinilbenzeno (DVB).

2.7 Curvas de titulação de três diferentes resinas de troca catiônica: $A$, Resina sulfônica fortemente ácida em $1 \mathrm{M}$ de $\mathrm{KCl}$, grupo funcional $\mathrm{SO}_{3} \mathrm{H}$ (Amberlite IR-120). B, Resina fosfonica em $1 \mathrm{M}$ de $\mathrm{NaCl}$, grupo funcional $\mathrm{PO}_{3} \mathrm{H}_{2}$. C, Resina carboxílica em $1 \mathrm{M}$ de $\mathrm{KCl}$, grupo funcional $\mathrm{COOH}$ (Amberlite IRC-50).

2.8 Curvas esquemáticas de titulação para as soluções $\mathrm{NaOH}-\mathrm{NaNO}_{3}$ (A) com e (B) sem resina de troca iônica (IRC-84).

2.9 Processo de inchamento da resina de troca iônica.

3.1 Eletrodo combinado de vidro.

3.2 Partículas da resina trocadora de íons fracamente ácida C104E, e estrutura química.

3.3 (a) Titulador automático do Departamento de Química da PUCRio. (b) Titulação em batelada ou batch. (c) Medida do pH.

3.4 Curvas de titulação em batelada da resina para as três concentrações de sal.

3.5 Curvas de titulação com e sem resina para as três concentrações de sal: (a) $\mathrm{NaCl} 1 \mathrm{M}$, (b) $\mathrm{NaCl} 0,1 \mathrm{M}$, (c) $\mathrm{NaCl} 0,01 \mathrm{M}$.

3.6 Miliequivalentes de hidrogênio liberados nas curvas de titulação da resina para as três concentrações de sal.

3.7 Validação dos dados de $\mathrm{pH}$ calculados pelo HYPERQUAD, $\mathrm{NaCl}$ $0,01 \mathrm{M}$ - Sistema sem considerar a formação da espécie RCOONa.

3.8 Validação dos dados de pH calculados pelo HYPERQUAD, $\mathrm{NaCl}$ $0,01 \mathrm{M}$ - Sistema considerando as espécies $\mathrm{RCOOH}$ e $\mathrm{RCOONa}$.

3.9 Validação dos dados de $\mathrm{pH}$ calculados pelo HYPERQUAD, $\mathrm{NaCl}$ $0,1 \mathrm{M}$ - Sistema considerando as espécies $\mathrm{RCOOH}$ e RCOONa.

3.10 Validação dos dados de $\mathrm{pH}$ calculados pelo HYPERQUAD, $\mathrm{NaCl} 1$ $\mathrm{M}$ - Sistema considerando as espécies $\mathrm{RCOOH}$ e RCOONa.

3.11 Espectro de infravermelho da resina Purolite C104E.

3.12 Equipamento de microscopia eletrônica de varredura utilizado.

3.13 Imagens de microscopia eletrônica de varredura da resina: (a) superfície e (b) seção transversal.

3.14 Processo de medida do inchamento da resina. (a-c) Centrifugação. (d) Secagem a vácuo. 
3.15 Valores experimentais do inchamento mássico da resina para cada curva de titulação.

4.1 Representação da dupla camada elétrica segundo Stern. (a) Distribuição iônica na solução e na superfície em forma de camadas, sendo esta última representada com grupos carregados carboxila. (b) Comportamento do potencial elétrico, $\Psi$ e (c) distribuição de carga com a distância desde a superfície.

4.2 Representação dos domínios microscópicos do modelo.

4.3 Sequência dos domínios estruturais de um meio poroso espacialmente periódico tendendo ao limite homogeneizado $(\varepsilon \rightarrow 0)$.

4.4 Representação do domínio $\varepsilon$-periódico $\Omega^{\varepsilon}$ constituído pela repetição da célula elementar de domínio $Y^{\varepsilon}$.

4.5 Modelo de Placas planas paralelas na escala: (a) macroscópica e (b) microscópica.

4.6 Microestrutura da célula periódica em forma de placas planas paralelas.

5.1 Discretização do domínio em $\mathrm{Ne}$ elementos finitos lineares e funções de interpolação de suporte compacto.

6.1 Condições experimentais na determinação do inchamento (Inchamento livre).

6.2 Diagrama esquemático do algoritmo adotado para o cálculo do espaçamento meio entre as placas $H$.

6.3 Curvas de inchamento para os valores de $\mathrm{K}_{1}$ e $\mathrm{K}_{2}$ obtidos por HYPERQUAD (Tabela 6.1). Experimento (linhas tracejadas) e Modelo de PB (linhas sólidas), $E_{s}=2,5 \mathrm{MPa}, \delta=1 \times 10^{-9} \mathrm{~m}, A s=1600 \mathrm{~m}^{2} / \mathrm{g} . \quad 125$

6.4 Efeito do valor de $\mathrm{K}_{1}$ no Inchamento. $\mathrm{NaCl} 0,1 \mathrm{M}, E_{s}=2,5 \mathrm{MPa}$, $\mathrm{K}_{2}=5,1963 \mathrm{M}^{-1}, \delta=1 \times 10^{-9} \mathrm{~m}, A s=1600 \mathrm{~m}^{2} / \mathrm{g}$.

6.5 Efeito do valor de $\mathrm{K}_{2}$ no Inchamento. $\mathrm{NaCl} 0,1 \mathrm{M}, E_{s}=2,5 \mathrm{MPa}$, $\mathrm{K}_{1}=6,4669 \times 10^{-6} \mathrm{M}, \delta=1 \times 10^{-9} \mathrm{~m}$, As $=1600 \mathrm{~m}^{2} / \mathrm{g}$.

6.6 Efeito do valor do módulo de Young no Inchamento - $\mathrm{NaCl} 0,1$ M. Valores de $\mathrm{K}_{1}$ e $\mathrm{K}_{2}$ obtidos por HYPERQUAD, $\delta=1 \times 10^{-9} \mathrm{~m}$, $A s=1600 \mathrm{~m}^{2} / \mathrm{g}$.

6.7 Efeito do valor da área superficial específica no Inchamento - $\mathrm{NaCl}$ $0,1 \mathrm{M}$. Valores de $\mathrm{K}_{1}$ e $\mathrm{K}_{2}$ obtidos por HYPERQUAD, $E_{s}=3,2 \mathrm{MPa}$, $\delta=1 \times 10^{-9} \mathrm{~m}$.

6.8 Distância media entre as placas - $\mathrm{NaCl} 0,1 \mathrm{M}$. Valores de $\mathrm{K}_{1}$ e $\mathrm{K}_{2}$ obtidos por HYPERQUAD, $E_{s}=3,2 \mathrm{MPa}, \mathrm{As}=900 \mathrm{~m}^{2} / \mathrm{g}$.

6.9 Efeito do valor da espessura da placa no Inchamento - $\mathrm{NaCl} 0,1$ $\mathrm{M}$. Valores de $\mathrm{K}_{1}$ e $\mathrm{K}_{2}$ obtidos por HYPERQUAD, $\mathrm{E}_{s}=3,2 \mathrm{MPa}$, $\mathrm{As}=900 \mathrm{~m}^{2} / \mathrm{g}$.

6.10 Variação da densidade de carga superficial $\left(\sigma^{0}\right)$ e da concentração do bulk $\left(C_{b}\right)$ em função do $\mathrm{pH}-\mathrm{NaCl} 1 \mathrm{M}$. Modelo de PB, Valores de $\mathrm{K}_{1}$ e $\mathrm{K}_{2}$ obtidos por HYPERQUAD, $E_{s}=3,2 \mathrm{MPa}, \mathrm{As}=900 \mathrm{~m}^{2} / \mathrm{g}$, $\delta=1 \times 10^{-9} \mathrm{~m}$. 
6.11 Variação da densidade de carga superficial $\left(\sigma^{0}\right)$ e da concentração do bulk $\left(C_{b}\right)$ em função do $\mathrm{pH}-\mathrm{NaCl}$ 0,1 M. Modelo de PB, Valores de $\mathrm{K}_{1}$ e $\mathrm{K}_{2}$ obtidos por HYPERQUAD, $E_{s}=3,2 \mathrm{MPa}, \mathrm{As}=900 \mathrm{~m}^{2} / \mathrm{g}$, $\delta=1 \times 10^{-9} \mathrm{~m}$.

6.12 Variação da densidade de carga superficial $\left(\sigma^{0}\right)$ e da concentração do bulk $\left(C_{b}\right)$ em função do $\mathrm{pH}-\mathrm{NaCl} 0,01 \mathrm{M}$. Modelo de $\mathrm{PB}$, Valores de $\mathrm{K}_{1}$ e $\mathrm{K}_{2}$ obtidos por HYPERQUAD, $E_{s}=3,2 \mathrm{MPa}$, As $=900 \mathrm{~m}^{2} / \mathrm{g}, \delta=1 \times 10^{-9} \mathrm{~m}$.

A.1 Efeito do valor de $\mathrm{K}_{1}$ no Inchamento. $\mathrm{NaCl} 1 \mathrm{M}, E_{s}=2,5 \mathrm{MPa}$, $\mathrm{K}_{2}=0,8482 \mathrm{M}^{-1}, \delta=1 \times 10^{-9} \mathrm{~m}, A s=1600 \mathrm{~m}^{2} / \mathrm{g}$.

A.2 Efeito do valor de $\mathrm{K}_{1}$ no Inchamento. $\mathrm{NaCl} 0,01 \mathrm{M}, E_{s}=2,5 \mathrm{MPa}$, $\mathrm{K}_{2}=0,6987 \mathrm{M}^{-1}, \delta=1 \times 10^{-9} \mathrm{~m}, A s=1600 \mathrm{~m}^{2} / \mathrm{g}$.

A.3 Efeito do valor de $\mathrm{K}_{2}$ no Inchamento. $\mathrm{NaCl} 1 \mathrm{M}, E_{s}=2,5 \mathrm{MPa}$, $\mathrm{K}_{1}=6,739 \times 10^{-7} \mathrm{M}, \delta=1 \times 10^{-9} \mathrm{~m}, A s=1600 \mathrm{~m}^{2} / \mathrm{g}$.

A.4 Efeito do valor de $\mathrm{K}_{2}$ no Inchamento. $\mathrm{NaCl} 0,01 \mathrm{M}, E_{s}=2,5 \mathrm{MPa}$, $\mathrm{K}_{1}=9,8084 \times 10^{-6} \mathrm{M}, \delta=1 \times 10^{-9} \mathrm{~m}, A s=1600 \mathrm{~m}^{2} / \mathrm{g}$.

A.5 Efeito do valor do módulo de Young no Inchamento - $\mathrm{NaCl} 1$ M. Valores de $\mathrm{K}_{1}$ e $\mathrm{K}_{2}$ obtidos por HYPERQUAD, $\delta=1 \times 10^{-9} \mathrm{~m}$, As $=1600 \mathrm{~m}^{2} / \mathrm{g}$.

A.6 Efeito do valor do módulo de Young no Inchamento - $\mathrm{NaCl} 0,01$ M. Valores de $\mathrm{K}_{1}$ e $\mathrm{K}_{2}$ obtidos por HYPERQUAD, $\delta=1 \times 10^{-9} \mathrm{~m}$, $A s=1600 \mathrm{~m}^{2} / \mathrm{g}$.

A.7 Efeito do valor da área superficial no Inchamento - $\mathrm{NaCl} 1 \mathrm{M}$. Valores de $\mathrm{K}_{1}$ e $\mathrm{K}_{2}$ obtidos por HYPERQUAD, $E_{s}=3,2 \mathrm{MPa}$, $\delta=1 \times 10^{-9} \mathrm{~m}$.

A.8 Efeito do valor da área superficial no Inchamento - $\mathrm{NaCl} 0,01$ M. Valores de $\mathrm{K}_{1}$ e $\mathrm{K}_{2}$ obtidos por HYPERQUAD, $E_{s}=3,2 \mathrm{MPa}$, $\delta=1 \times 10^{-9} \mathrm{~m}$.

A.9 Efeito do valor da espessura da placa no $H-\mathrm{NaCl} 1 \mathrm{M}$. Valores de $\mathrm{K}_{1}$ e $\mathrm{K}_{2}$ obtidos por HYPERQUAD, $E_{s}=3,2 \mathrm{MPa}, \mathrm{As}=900 \mathrm{~m}^{2} / \mathrm{g}$.

A.10 Efeito do valor da espessura da placa no Inchamento - $\mathrm{NaCl} 1$ M. Valores de $\mathrm{K}_{1}$ e $\mathrm{K}_{2}$ obtidos por HYPERQUAD, $E_{s}=3,2 \mathrm{MPa}$, As $=900 \mathrm{~m}^{2} / \mathrm{g}$.

A.11 Efeito do valor da espessura da placa no $H-\mathrm{NaCl} 0,01 \mathrm{M}$. Valores de $\mathrm{K}_{1}$ e $\mathrm{K}_{2}$ obtidos por HYPERQUAD, $E_{s}=3,2 \mathrm{MPa}$, As $=900 \mathrm{~m}^{2} / \mathrm{g} .158$

A.12 Efeito do valor da espessura da placa no Inchamento - $\mathrm{NaCl} 0,01$ M. Valores de $\mathrm{K}_{1}$ e $\mathrm{K}_{2}$ obtidos por HYPERQUAD, $E_{s}=3,2 \mathrm{MPa}$, $\mathrm{As}=900 \mathrm{~m}^{2} / \mathrm{g}$. 


\section{Lista de tabelas}

2.1 Características de alguns tipos de resinas comerciais disponíveis no mercado.

3.1 Concentrações das soluções empregadas nas titulações em batelada da resina.

3.2 Valores de capacidade de troca catiônica (CTC) da resina correspondentes a cada titulação em batelada.

3.3 Constantes de formação e de equilíbrio obtidas mediante HYPERQUAD para as três titulações

3.4 Constantes de equilíbrio reportadas para polímeros e resinas de troca iônica carboxílicas

6.1 Constantes de equilíbrio obtidas mediante HYPERQUAD. 
Que tus ojos se convenzan de la suerte que tuvimos de tener madera fina.. no se parte.. se hace fuerte con el tiempo.. Vida de madera fina, no se pierde en la tormenta, si la tumban se levanta.. hechos con sudor y tierra, alma y corazón despiertos, Somos de madera fina..

Giordano Di Marzo, Finales de Siglo. 